\title{
Modeling Coherent Anti-Stokes Raman Scattering with Time-Dependent Density Functional Theory: Vacuum and Surface Enhancement
}

\section{Citation}

Parkhill, John A., Dmitrij Rappoport, and Alan Aspuru-Guzik. 2011. Modeling coherent antistokes raman scattering with time-dependent density functional theory: Vacuum and surface enhancement. Journal of Physical Chemistry Letters 2(15): 1849-1854.

\section{Published Version}

doi:10.1021/jz2005573

\section{Permanent link}

http://nrs.harvard.edu/urn-3:HUL.InstRepos:8365121

\section{Terms of Use}

This article was downloaded from Harvard University's DASH repository, and is made available under the terms and conditions applicable to Open Access Policy Articles, as set forth at http:// nrs.harvard.edu/urn-3:HUL.InstRepos:dash.current.terms-of-use\#OAP

\section{Share Your Story}

The Harvard community has made this article openly available.

Please share how this access benefits you. Submit a story.

\section{Accessibility}




\title{
Modeling Coherent Anti-Stokes Raman Scattering
}

\section{with Time-Dependent Density Functional Theory:}

\section{Vacuum and Surface Enhancement.}

\author{
John A. Parkhill, ${ }^{*}$ Dmitrij Rappoport, ${ }^{*}$ and Alan Aspuru-Guzik* \\ Department of Chemistry and Chemical Biology, Harvard University, 12 Oxford St. Cambridge, \\ MA 02138, USA \\ E-mail: john.parkhill@gmail.com; rappoport@chemistry.harvard.edu; \\ aspuru@chemistry.harvard.edu
}

*To whom correspondence should be addressed 


\begin{abstract}
We present the first density functional simulations of coherent anti-Stokes Raman scattering (CARS), and an analysis of the chemical effects upon binding to a metal surface. Spectra are obtained from first-principles electronic structure calculations, and are compared with available experiments and previously available theoretical results following from Hartree-Fock polarizability derivatives. A first approximation to the non-resonant portion of the CARS signal is also explored. We examine the silver-pyridine cluster models of the surface chemical signal enhancement, previously introduced for surface enhanced Raman scattering. Chemical resonant intensity enhancements of roughly $10^{2}$ are found for several model clusters. The prospects of realizing further enhancement of CARS signal with metal surfaces is discussed in light of the predicted chemical enhancements. Keywords: TDDFT, CARS, Raman, Chemical Enhancement
\end{abstract}


Coherent anti-Stokes Raman Scattering $(\mathrm{CARS})^{1}$ is an increasingly popular four-wave mixing optical technique for spectroscopy and chemically specific microscopy. The advantages offered over simple spontaneous Raman spectroscopy are the coherent signal blue-shifted away from fluorescence, and rapid acquisition from the collective response of many chromophores. Recent advances in the microscopic applications of this technique have improved signal collection through the use of tightly focused beams ${ }^{2}$ which relax the phase matching condition, and techniques for limiting the non-resonant signal. ${ }^{3,4}$ Spectroscopic studies have separated resonant from non-resonant signal in the time-domain ${ }^{3}$ and designed more efficient ${ }^{5,6}$ collection sequences. The enhancement of CARS by intense plasmon fields could potentially enhance signal further and is the motivation for this work. In order to address this problem theoretically we have developed code to simulate CARS spectra of molecules bound to cluster models of metal surfaces which is described below.

The enhancement of spectroscopies by the intense fields of nearby collective excitations in metals or semiconductors has experienced a renaissance in recent years, especially surface-enhanced Raman scattering (SERS). ${ }^{7}$ Given the non-linear dependence of the CARS signal on input power $\left(I_{\mathrm{CARS}} \propto E_{1}^{2} E_{2}\right)$ one might expect surface-enhanced CARS (SE-CARS). The first report of CARS occurring near a surface plasmon was published decades ago, ${ }^{8}$ and followed by reports of CARS observed from single molecules, ${ }^{9}$ and on the tips of scanning-tunneling microscopes. ${ }^{10}$ Despite these advances, the paucity of SE-CARS spectra, and scattering cross-sections available in the literature indicate that this remains a difficult experiment to perform and completely characterize. We will try to offer some indication of chemical enhancement effects of SE-CARS, and in the last section point out challenges facing application of the spectroscopy which should be given further attention.

Past theory has focused ${ }^{11,12}$ on understanding the classical electrodynamic features of CARS spectroscopy until Hartree-Fock(HF) polarizability derivatives were combined with (static) density functional theory (DFT) vibrational frequencies to produce the first ab-initio CARS spectra. ${ }^{13,14}$ HF theory's neglect of electron-electron interaction is especially unphysical for systems with sig- 
nificant correlation, like metals. For this reason, we replace HF for the calculation of CARS on metal cluster models. In this work we present the first CARS spectra calculated entirely with DFT. Prior to applying the method to metal clusters we test the model on a few known experimental spectra from previous work to make some estimation of its usefulness.

\section{Theory}

The CARS spectra in this paper are calculated in the double-harmonic approximation developed by the Aarhus group, ${ }^{13}$ which follows from the much older double harmonic approximation to Raman spectra, ${ }^{15}$ which we briefly review. The typical CARS experiment applies two lasers of frequencies $\omega_{1}, \omega_{2}$ which scatter twice and once respectively off the target material. Signal radiation collected at $2 \omega_{1}-\omega_{2}$ peaks when the Stokes shift, $\omega_{1}-\omega_{2}$ coincides with a vibrational transition of the scatterer. On the scale of a single molecule CARS is governed by the second hyperpolarizabiliity $\left(\chi^{(3)}\left(-2 \omega_{1}-\omega_{2} ; \omega_{1},-\omega_{2}, \omega_{1}\right)\right)$. We include terms where the central resolution in the sum-over-states expression for $\chi^{(3)}$ only includes excited vibrational states on the electronic ground state because these are resonant with the wavelength differences of the experiment. $\chi^{(3)}$ is then a product of polarizabilities, albeit with perturbed nuclear geometries. Expanding the polarizability as a power series to first order along each mode, as in the harmonic approximation for Raman cross sections the expression for the resonant CARS susceptibility becomes:

$$
\begin{aligned}
\chi_{R}^{(3)}{ }_{(i j k l)} \approx & \frac{2}{\hbar} \sum_{n} \frac{d \alpha_{i j}\left(\omega_{1}\right)}{d Q_{n}} \frac{d \alpha_{k l}\left(\omega_{1}\right)}{d Q_{n}}\left(\omega_{n}-\left(\omega_{1}-\omega_{2}\right)-i \varepsilon\right)^{-1}+ \\
& \frac{2}{\hbar} \sum_{n} \frac{d \alpha_{i l}\left(\omega_{1}\right)}{d Q_{n}} \frac{d \alpha_{k j}\left(\omega_{1}\right)}{d Q_{n}}\left(\omega_{n}+\left(\omega_{1}-\omega_{2}\right)+i \varepsilon\right)^{-1}
\end{aligned}
$$

The formula above incorporates some simplifications emerging from spherical averaging ${ }^{16}$ ex- $^{-}$ plained in the supporting information. Here $\varepsilon$ is a phenomenological linewidth (inverse lifetime), $\{i j k l\}$ are Cartesian indices, and $Q_{n}$ are nuclear displacements along mode $n$. 


\section{Non-Resonant Appoximation}

There is also a contribution to the CARS signal which is non-resonant(NR) with any of the applied frequencies or their differences, $\chi_{N R}^{(3)}$ which we discuss below. Including this term, and averaging over the molecular orientations the expression for the CARS signal in an isotropic medium becomes:

$$
I_{\mathrm{CARS}} \propto\left|\left(\bar{\chi}_{R}^{(3)}+\bar{\chi}_{N R}^{(3)}\right)\right|^{2} ; \text { where } \bar{\chi}^{(3)}=\frac{1}{15} \sum_{i j}\left(\chi_{i j j i}^{(3)}+\chi_{i j i j}^{(3)}+\chi_{i i j j}^{(3)}\right)
$$

The single-scatterer expression given above ignores the coherent amplification from many molecules in an illuminated volume $\left(d^{3}\right.$, where $\mathrm{d}$ is the diameter of the coherent volume), $I_{\mathrm{CARS}} \propto d^{2}$ which is experimentally vital to realize intense signal especially in microscopy. ${ }^{17}$ Also note the dimensions of a molecular polarizability are different from the macroscopic property, and although one may convert between them approximately. This ignores local-field effects and has not pursued here. Although $\chi_{N R}^{(3)}$ is nearly constant in regions of interest, it contributes to the observed fourwave mixing signal and distorts it in the vicinity of resonances of $\chi^{(3)}$ because of the cross term in Eq. 2. We will consider only the electronic contributions to this signal, which for sequence of applied fields we are interested in, breaks into two diagrams. ${ }^{18}$ The first resembles the resonant CARS diagram, but sums over electronically excited states. The second resembles a two-photon absorption-emission process, and usually becomes dominant at high pump energy, where $2 \omega_{1}$ is near electronic resonance. The relative magnitude of the non-resonant signal varies significantly ${ }^{3}$ with small changes in the pulse sequence and other factors such as light polarization, ${ }^{19}$ because the non-resonant electronic dynamics is very fast on the vibrational timescale. To obtain quantitative simulation, one needs precise characterization of the experiments, and this is one reason for the infrequent appearance of absolute CARS cross sections in the literature. We are interested in how well the non-resonant contribution could be modeled by the first diagram, that is $\chi_{e}^{(3)} \approx \chi^{(1)}\left(\omega_{1}\right) \chi^{(1)}\left(\omega_{1}\right) / \omega_{1}$. This approximation is admittedly very rough, but desirably efficient. 
We will use this approximation to the non-resonant signal chiefly to obtain spectra in qualitative agreement with those available from the literature and verify that DFT and TDDFT can be usefully paired to simulate CARS spectra. Approximations of this sort were made long ago by Wang, ${ }^{20}$ and are appropriate when the applied radiation is far below resonance $\left(2 \omega \hbar<<\left(E_{1}-E_{0}\right)\right)$, where $E_{i}$ are energies of adiabatic electronic states). This condition roughly holds for the organic species studied here where $\sim 700 \mathrm{~nm}$ light is applied. The finite timescale of the electronic scattering process can also be represented by the imaginary part of these quantities. ${ }^{21}$ The non-resonant signal from a metal nano-particle is expected to dominate the background of SE-CARS, and depend on excitations spatially extended beyond the range of a TDDFT simulation. Nanoscale simulations of four-wave mixing are not well developed, and interfacing those with TDDFT would be an undertaking beyond the scope of this work. For the purposes of surface chemical enhancement we will not include non-resonant signal.

\section{Performance of TDDFT}

In the case of spontaneous Raman DFT is known to provide accurate spectra, ${ }^{22,23}$ but to establish the usefulness of TDDFT polarizability derivatives for CARS we compare them here with those from TDHF, and experiment. ${ }^{24,25}$ The spectra of anthracene and perylene are shown below in Figure 1 and Figure 2 respectively. For anthracene (Fig. Figure 1), the non-resonant approximation achieves a reasonable distortion which matches the experimental lineshape in a satisfactory way. The relative phasing of each peak can vary because of the anisotropy of $X_{N R}^{(3)}$. The peak intensities are well reproduced with the exception of the mode at $1590 \mathrm{~cm}^{-1}$ which is exaggerated, as was seen in previous work. ${ }^{13}$ Motion along this mode lengthens the bonds between the tertiary carbons, stretching the short axis of the molecule. The spectrum of perylene (Fig. Figure 2) is again, roughly accurate, although the relative intensities of the two important peaks are switched. Comparing against the spectra from ref. ${ }^{13}$ and knowing that the perylene has a low-lying first excited state at roughly $24,000 \mathrm{~cm}^{-126}$ these are likely artifacts of our approximation to the NR signal. 
The exaggeration of the first peak height is more severe in the TDDFT than in the HF results, and the distortion due to the NR signal is significantly different in the HF and B3LYP spectra. Going back to the polarizabilities themselves, TDDFT predicts the $\alpha_{x x}$ component, the long-axis along which perylene is oriented, to be a factor of two larger than HF.

Many common functionals are known to exaggerate polarizabilities ${ }^{27}$ of delocalized systems,

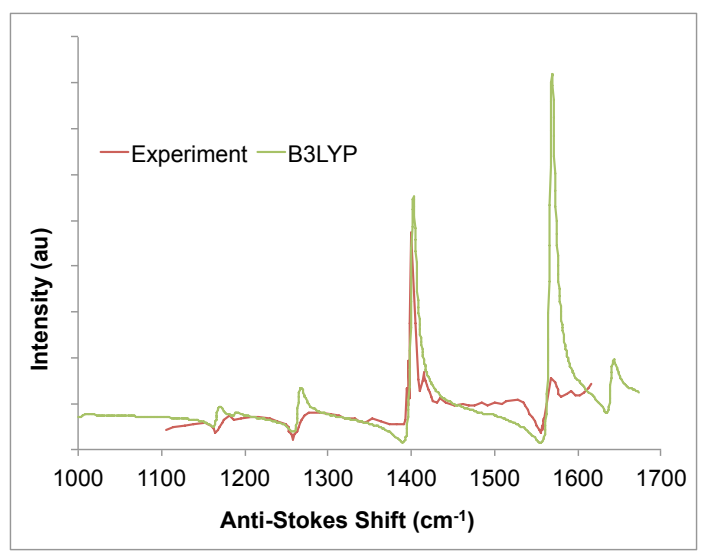

Figure 1: A comparison of experimental anthracene CARS spectra with those obtained from TDDFT simulations. Agreement is satisfactory with the exception of the mode near $1560 \mathrm{~cm}^{-} 1$

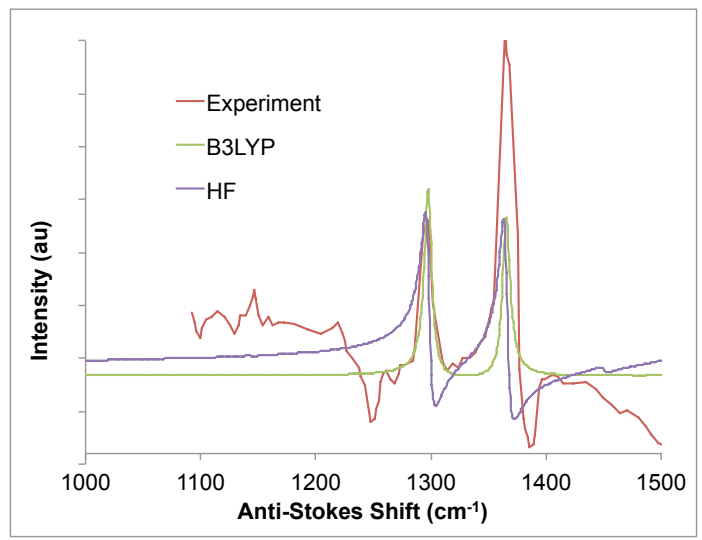

Figure 2: TDDFT and Experimental Perylene CARS spectra. The approximation to nonresonant signal employed is compromised by a low-lying first excited state, but there is still semiquantitative agreement.

due to the incorrect asymptotic behavior of the local density approximation exchange potential. Roughly speaking, the CARS intensity in the resonant approximation should go as the square of 
this quantity. Although the B3LYP functional seems sufficient for these hydrocarbons which extend in space as far as the metal clusters we will examine in the next section, an asymptotically correct exchange functional ${ }^{28}$ would be desirable for studies of larger molecules. The lack of correlation effects in HF may favor TDDFT for a metal cluster study.

\section{Surface Effects}

Metal surfaces largely enhance (spontaneous) Raman spectra via the increased fields which exist in the vicinity of a surface plasmon excitation, ${ }^{29-31}$ although there is also a chemical effect ${ }^{32-35}$ which arises from the metal perturbing the electronic structure of an attached molecule. Classical electrodynamics can account for the former, ${ }^{36}$ and TDDFT calculations can be used to describe the latter. ${ }^{34,35,37}$ Recent algorithmic developments have brought both theories into a single selfconsistent calculation, ${ }^{38}$ and also provided a rigorous quantum treatment ${ }^{39}$ of the the matter-field interaction in a surface-enhanced Raman scattering event. Large $\left(\sim 10^{12}\right)$ enhancement factors 40 for SE-CARS were predicted decades ago on the basis of classical, electromagnetic Mie theory, although experiments have not realized anything comparable. Enhancements of $\sim 10^{3}$ have been reported, ${ }^{41}$ but are not common. SE-CARS remains an ongoing pursuit.

Based on the the SERS precedent, a cluster-model CARS spectra should represent the chemical effects of the bulk. The small molecule is coupled to metallic states, but to a first approximation the electronic states with significant density on the small molecule remain local enough to fit within the cluster. Thus the polarizability gradients responsible for resonant signal should approximately depend on the electronic structure only near the small molecule, and our DFT/TDDFT approach can be used to examine chemical enhancement of CARS. A CARS experiment for pyridine on silver colloid has been recently published ${ }^{42}$ which reports a 10x CARS signal enhancement, and a SERS enhancement of $10^{4}$. Cluster models have been systemically investigated ${ }^{43}$ for simulating the chemical portion of the enhancement. We use the well-known silver-pyramid cluster-model ${ }^{44}$ which was also used for a recent surface- enhanced Hyper-Raman scattering (SEHRS) simulation 
of this system. ${ }^{33}$ Some additional clusters were also optimized for this work (see supporting information), with the expectation that chemical enhancement of CARS would more significantly vary than for SERS. Indeed they do vary, although the trends can be understood. The structures employed are summarized in Figure Figure 3, and given in supporting information.

The CARS spectrum of an isolated pyridine molecule (Figure 4) already appears significantly

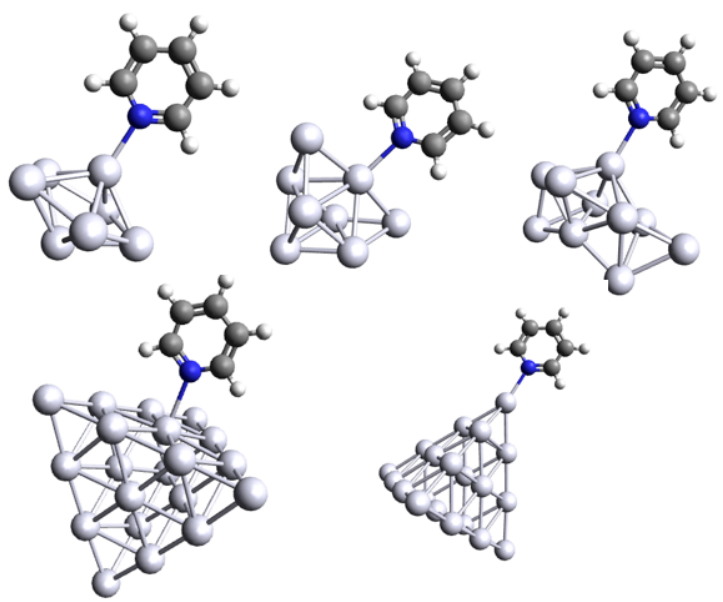

Figure 3: Silver-Pyridine cluster models employed to simulate SE-CARS. Left-to-right: $\{7,8,10$, $20 \mathrm{~S}, 20 \mathrm{~V}$ \}. For those which posses an even number of electrons, restricted calculations have been employed.

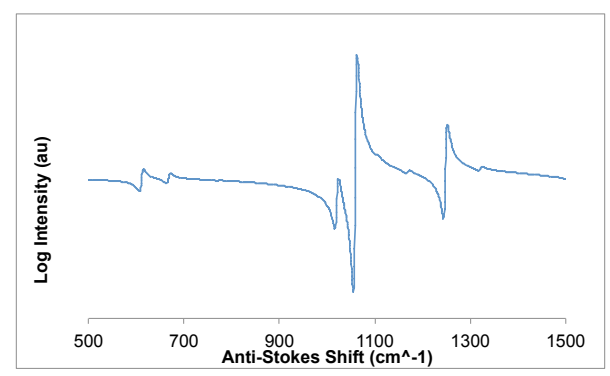

Figure 4: Simulated (PBE0/TZVP) pyridine CARS spectra.

distorted by non-resonant contributions especially the bright peaks near $1030 \mathrm{~cm}^{-1}$ in accordance with some available spectra. ${ }^{45}$ The resonant enhancement of bright peaks $\left(I_{\text {pyr-Ag }} / I_{\text {pyr }}\right)$ relative to the isolated pyridine spectrum are tabulated in Table (Table 1) for the clusters examined. Whether DFT or HF polarizabilities are used the enhancement trends are found to be quite similar. The 
ring-breathing mode of pyridine at $611 \mathrm{~cm}^{-1}$ stretches the ring pulling the nitrogen atom away from the para carbon on the other side of the ring, and is strongly enhanced by the presence of the metal surface. The most strongly enhanced peaks near $1020 \mathrm{~cm}^{-1}$ and $1058^{-1}$ also correspond to ring distortions which involve the $\mathrm{Ag}-\mathrm{N}$ bond. The experimental ${ }^{42} \mathrm{SE}-\mathrm{CARS}$ spectrum between 700 and $1400 \mathrm{~cm}^{-1}$ shows two broad, structured peaks centered near $1000 \mathrm{~cm}^{-1}$ and $1150 \mathrm{~cm}^{-1}$. As in our computational results the peak near $1000 \mathrm{~cm}^{-1}$ is brightest. Detailed discussion is given in the supporting information.

Like other SE Raman spectroscopies, the chemical enhancement can be understood from a simplified picture of the changes the electronic system undergoes when the substrate is brought into contact with the metal. ${ }^{46-48}$ Following previous that previous work, resonant signal in Eq.(1) should be proportional to the square of the Raman polarizability derivative, $\left|\left(\frac{d \alpha_{i j}}{d Q_{k}}\right)^{2}\right|^{2}$. The polarizability itself in the low-energy region we are interested in is often dominated by a low-lying bright state of energy $\omega_{e}$. Taking the static limit, and ignoring changes in the transition moments along modes, $\mu_{\alpha}=\left\langle e\left|\mu_{\alpha}\right| 0\right\rangle$, gives a two-state expression for the derivative: $\frac{d \alpha_{\alpha \beta}}{d Q_{k}} \approx-\frac{\mu_{\alpha} \mu_{\beta}}{\omega_{e}^{2}} \frac{d \omega_{e}}{d Q_{k}}$. This analysis suggests that the ratio of the signal from a single adsorbed species to a free species goes as $I_{\mathrm{Ag}-\mathrm{Pyr}} / I_{\mathrm{Pyr}} \propto\left(\frac{\omega_{\mathrm{Pyr}}}{\omega_{\mathrm{Ag}-\mathrm{Pyr}}}\right)^{8}$, where the $\omega$ is an estimate of the lowest bright transition energy in the respective species. The proportionality constant absorbs the ratio of adsorbed and free transition moments, and $\frac{d \omega_{e}}{d Q_{k}}$. Estimating $\omega_{\mathrm{Pyr}}$ as the occupied-unoccupied gap, and $\omega_{\mathrm{Pyr}-\mathrm{Ag}}$ as the difference between the Ag HOMO and energy of the pyridine LUMO in the cluster, the Ag-7, 20S and 20V enhancement factors are estimated to be 6,416 , and 701. The integrated static enhancements obtained from the ab-initio calculations are: 4, 559, and 1,070, roughly the correct order of magnitude and the correct trend.

We predict a chemical contribution to the enhancement of the CARS signal which is substantial on the scale of observed overall enhancements. Given the certain dominance of the EM mechanism in SERS, ${ }^{7}$ this suggests that our single-molecule results are only one ingredient of a more nuanced experimental picture. In the next section we propose how this may be investigated by experiment and theory. 
Table 1: Relative enhancements of significant CARS peaks on cluster models, frequencies in $\mathrm{cm}^{-1}$. HF results are relative to a pyridine spectrum from Hartree-Fock polarizability derivatives others from PBE0 (results from the resonant approximation).

\begin{tabular}{c||c|c|c|c|c|c|c}
\hline \hline Vib Freq. & $\mathrm{Ag}_{7}$ & $\mathrm{Ag}_{8}$ & $\mathrm{Ag}_{10}$ & $\mathrm{Ag}_{20}(\mathrm{~s})$ & $\mathrm{Ag}_{20}(\mathrm{v})$ & $\mathrm{Ag}_{20}(\mathrm{~s})(\mathrm{HF})$ & $\mathrm{Ag}_{20}(\mathrm{v})(\mathrm{HF})$ \\
\hline \hline 611 & 39.9 & 1.9 & 445.8 & 46.5 & 0.3 & 205.9 & 0.1 \\
667 & 0.0 & 0.1 & 13.6 & 0.4 & 0.4 & 1.0 & 0.3 \\
1020 & 78.7 & 4.0 & 243.8 & 681.0 & 348.7 & 570.7 & 165.7 \\
1058 & 25.2 & 0.1 & 0.9 & 12.3 & 128.0 & 1.5 & 22.4 \\
1247 & 2.5 & 25.2 & 4.5 & 1.3 & 17.4 & 2.1 & 35.4 \\
1648 & 0.0 & 7.2 & 35.6 & 1.0 & 1.0 & 1.1 & 0.0 \\
1652 & 254.2 & 0.0 & 42.8 & 9.5 & 195.8 & 3.1 & 16.5 \\
\hline \hline
\end{tabular}

\section{Challenges facing SE-CARS}

SEHRS $^{33,49,50}$ is relatively better characterized than SE-CARS ${ }^{8,10,41,51,52}$ and higher hyperRaman enhancement factors have been realized, although both are non-linear spectroscopies of significant complexity. We would like to note three reasons this may be so. Previous authors have pointed out the complication of the phase-matching condition by a nearby metal surface, ${ }^{42}$ which can be addressed by adopting a tight-focusing experimental setup. A classical electromagnetic analysis of SE-CARS including these difficulties predicted enhancement factors of $10^{12} \cdot{ }^{40}$ The surfaces of noble metals are also known to conduct four-wave mixing ${ }^{53,54}$ which would mask the resonant signal of molecules. Given that this non-linear effect is the result of short lived scattering from electronic states of gold, it is possible to separate this background from resonant signal by pulse timing, ${ }^{3,55,56}$ polarization or shaping ${ }^{12}$ techniques.

The rapid dephasing of vibrational quanta on metal surfaces has not received much attention from the SE-CARS community, but warrants further study. Unlike spontaneous Raman, and hyperRaman, the resonant CARS wavepacket must pass through a vibrational coherence and interact with light again to contribute signal. This nuclear wave-packet may dephase so rapidly on a metal surface that the electromagnetically enhanced signal is reduced. Detailed pictures of the timedependence of the density matrix in a CARS experiment have been developed in the literature. ${ }^{57}$ The dependence of the CARS signal on vibrational dephasing been exploited in the past to make measurements of the pure dephasing rates. ${ }^{58,59}$ For a molecule like anthracene in a crystal, relax- 
ation lifetimes are on the order of 10ps. Plasmons readily accept energy from molecular vibrations and reduce $T_{1}$ relative to the vacuum to roughly $1 \mathrm{ps}$. This can be calculated from cluster models using the golden rule. ${ }^{60}$ The $T_{2}^{*}$ dephasing will have the usual collisional contributions, and also a component unique to SE-CARS from the vibrational transition dipole coupling to rapid fluctuations of the plasmon, and partially localized vibrational modes. A molecular dynamics simulation coupled to a properly fluctuating classical electric field, may be able to theoretically predict both pure-dephasing rates. ${ }^{61}$ Pump-Probe experiments to measure the SE-CARS dephasing timescale may also shed light on the situation. ${ }^{12,62}$

For the purposes of simulating SE-CARS, we have presented the first calculations of CARS spectra entirely with DFT. The hybrid functionals employed here provide accuracy comparable to $\mathrm{HF}$, and the conclusions drawn about chemical enhancement of CARS are qualitatively the same whether HF or DFT polarizability derivatives are employed. Approximating the dynamic electronic second hyperpolarizability as a separable product of the dynamic polarizability appears to offer satisfactory modeling of the non-resonant background signal for pump frequencies well below material electronic excitations. The bulk spectra provided with TDDFT are in semi-quantitative agreement with available experimental data.

Small cluster models are found to offer simulated SE-CARS spectra of pyridine on silver which are consistent whether HF or TDDFT polarizabilities are used. The most intense peaks of the available experiment are also the brightest peak in our simulations. Based on the results of cluster-model calculations we estimate the chemical enhancement of a pyridine-silver SE-CARS experiment to be significant when compared to available experimental information without any treatment of electromagnetic enhancement. Optimistically, this indicates a mechanism for metals to enhance CARS, but also suggests that a complete picture of SE-CARS will be more complex than SERS and likely require a treatment of dephasing. Future work should focus on the calculation of the non-resonant signal produced by the metal, perhaps via a classical electromagnetic simulation, although the inclusion of this signal in an "enhancement factor" may be ambiguous. We have pointed out three possible explanations for the difficulty of measuring SE-CARS: phase-matching, background sig- 
nal and dephasing. Future work will calculate the rate of vibrational dephasing to acertain whether the dephasing effects which are unique to a metal-molecule system are significant compared to chemical and electromagnetic enhancements. The other two complications: the non-resonant signal and phase matching, might be avoided with experimental effort. Pure-dephasing induced by the metal surface is however a nearly intrinsic part of the SE-CARS experiment.

\section{Computational Details}

The results of this paper were calculated within the Turbomole electronic structure package. ${ }^{63-65}$ The def2-TZVP basis ${ }^{66}$ was employed in all cases, with matching pseudo-potential for metallic elements. The geometries of organic species were optimized with the same basis, and the $\mathrm{B} 3 \mathrm{LYP}^{67}$ functional, the metal clusters were optimized and polarizability derivatives evaluated with the PBE0 ${ }^{68,69}$ functional. Further details can be obtained in the supporting information. An empirical inverse lifetime of $\varepsilon=2 \mathrm{~cm}^{-1}$ has been assumed for the calculations unless otherwise noted. Throughout the paper the label HF attached to a spectrum indicates that it was generated with HF polarizability derivatives calculated along the normal modes emerging from a B3LYP calculation, ie: the procedure which was previously available. ${ }^{13}$ Although note that in that work $\chi_{N R}^{(3)}=\chi_{e}^{(3)}$, whereas in this work it's approximated as the product of dynamic polarizabilities described above. No subsequent scaling of the vibrational frequencies of DFT has been performed.

\section{Acknowledgments}

This work was supported by the Defense Advanced Research Project Agency under Contract No .FA9550-08-1- 0285 and the Defense Threat Reduction Agency under Contract No. HDTRA110-1-0046. The authors thank Dr. Paul Peng, Dr. Semion Saikin and Roberto Olivares-Amaya for several useful discussions. A.A.G. thanks the Camille and Henry Dreyfus and Sloan foundations for their generous support.

Supporting Information Available: Supplemental equations, results, molecular geometries and 
detailed computational information are available. This material is available free of charge via the Internet at http://pubs.acs.org.

\section{References}

(1) Maker, P.; Terhune, R. Study of optical effects due to an induced polarization third order in the electric field strength. Phys. Rev. 1965, A137, 801.

(2) Evans, C. L.; Xie, X. S. Coherent Anti-Stokes Raman Scattering Microscopy: Chemical Imaging for Biology and Medicine. Ann. Rev. Anal. Chem. 2008, 1, 883-909.

(3) Compton, R.; Filin, A.; Romanov, D. A.; Levis, R. J. Elucidating the spectral and temporal contributions from the resonant and nonresonant response to femtosecond coherent antiStokes Raman scattering. J. Chem. Phys. 2008, 128, 154517.

(4) Roy, S.; Meyer, T. R.; Gord, J. R. Time-resolved dynamics of resonant and nonresonant broadband picosecond coherent anti-Stokes Raman scattering signals. Appl. Phys. Lett. 2005, $87,264103$.

(5) Knutsen, K.; Johnson, J.; Miller, A.; Petersen, P.; Saykally, R. High spectral resolution multiplex CARS spectroscopy using chirped pulses. Chem. Phys. Lett. 2004, 387, 436-441.

(6) Oron, D.; Dudovich, N.; Yelin, D.; Silberberg, Y. Narrow-Band Coherent Anti-Stokes Raman Signals from Broad-Band Pulses. Phys. Rev. Lett. 2002, 88, 063004.

(7) Schatz, G.; Duyne, R. V. Electromagnetic Mechanism of Surface-Enhanced Spectroscopy; Wiley, 2002; Vol. 1.

(8) Chen, C.; Castro, A. D.; Shen, Y.; DeMartini, F. Surface coherent anti-Stokes Raman spectroscopy. Phys. Rev. Lett. 1979, 43, 946-949.

(9) Koo, T.; Chan, S.; Berlin, A. Single-molecule detection of biomolecules by surface-enhanced coherent anti-Stokes Raman scattering. Opt. Lett. 2005, 30, 1024-1026. 
(10) Ichimura, T.; Hayazawa, N.; Hashimoto, M.; Inouye, Y.; Kawata, S. Tip-Enhanced Coherent Anti-Stokes Raman Scattering for Vibrational Nanoimaging. Phys. Rev. Lett. 2004, 92, 220801 .

(11) Yuratich, M.; Hanna, D. Coherent anti-Stokes Raman spectroscopy (CARS). Mol. Phys. 1977, 33, 671-682.

(12) Rahav, S.; Mukamel, S. Stimulated coherent anti-Stokes Raman spectroscopy (CARS) resonances originate from double-slit interference of two-photon Stokes pathways. Proc. Nat. Acad. Sci. 2010, 107, 4825-4829.

(13) Thorvaldsen, A. J.; Ferrighi, L.; Ruud, K.; Ågren, H.; Coriani, S.; Jørgensen, P. Analytic ab initio calculations of coherent anti-Stokes Raman scattering (CARS). Phys. Chem. Chem. Phys. 2009, 11, 2293.

(14) Mohammed, A.; Agren, H.; Thorvaldsen, A. J.; Ruud, K. Ab initio study of coherent antiStokes Raman scattering (CARS) of the 1,3,5-trinitro-1,3,5-triazacyclohexane (RDX) explosive. Chem. Phys. Lett. 2010, 485, $320-325$.

(15) Long, D. A. The Raman Effect; Wiley, 2002.

(16) Davis, R. P.; Moad, A. J.; Goeken, G. S.; Wampler, R. D.; Simpson, G. J. Selection Rules and Symmetry Relations for Four-Wave Mixing Measurements of Uniaxial Assemblies. The Journal of Physical Chemistry B 2008, 112, 5834-5848.

(17) Cheng, J.; Xie, X. Coherent anti-Stokes Raman scattering microscopy: instrumentation, theory, and applications. J. Phys. Chem. B 2004, 108, 827-840.

(18) Moad, A.; Simpson, G. Self-consistent approach for simplifying the molecular interpretation of nonlinear optical and multiphoton phenomena. J. Phys. Chem. A 2005, 109, 1316-1323.

(19) Cheng, J.-X.; Book, L. D.; Xie, X. S. Polarization coherent anti-Stokes Raman scattering microscopy. Opt. Lett. 2001, 26, 1341-1343. 
(20) Wang, C. C. Empirical Relation between the Linear and the Third-Order Nonlinear Optical Susceptibilities. Phys. Rev. B 1970, 2, 2045-2048.

(21) Jensen, L.; Autschbach, J.; Schatz, G. C. Finite lifetime effects on the polarizability within time-dependent density-functional theory. J. Chem. Phys. 2005, 122, 4115.

(22) Halls, M. D.; Schlegel, H. B. Comparison study of the prediction of Raman intensities using electronic structure methods. J. Chem. Phys. 1999, 111, 8819-8824.

(23) Zvereva, E. E.; Shagidullin, A. R.; Katsyuba, S. A. Ab Initio and DFT Predictions of Infrared Intensities and Raman Activities. J. Phys. Chem. A 2011, 115, 63-69.

(24) Hare, D. V.; Carreira, L. A.; Rogers, L.; Azarraga, L. Coherent Anti-Stokes Raman Spectroscopy of Polycyclic Aromatic Hydrocarbons. Appl. Spec. 1984, 38, 543-552.

(25) Carreira, L. A.; Maguire, T. C.; Malloy, T. B. Coherent anti-Stokes resonance Raman excitation profiles of tetracene. Spec. Acta 1986, 1-7.

(26) Halasinski, T. M.; Weisman, J. L.; Ruiterkamp, R.; Lee, T. J.; Salama, F.; Head-Gordon, M. Electronic Absorption Spectra of Neutral Perylene (C20H12), Terrylene (C30H16), and Quaterrylene (C40H20) and Their Positive and Negative Ions: Ne Matrix-Isolation Spectroscopy and Time-Dependent Density Functional Theory Calculations. The Journal of Physical Chemistry A 2003, 107, 3660-3669.

(27) Hammond, J. R.; Kowalski, K.; deJong, W. A. Dynamic polarizabilities of polyaromatic hydrocarbons using coupled-cluster linear response theory. The Journal of Chemical Physics 2007, 127, 144105.

(28) Silverstein, D. W.; Jensen, L. Assessment of the accuracy of long-range corrected functionals for describing the electronic and optical properties of silver clusters. The Journal of Chemical Physics 2010, 132, 194302.

(29) Moskovits, M. Surface-enhanced spectroscopy. Rev. Mod. Phys. 1985, 57, 783-826. 
(30) Kneipp, K.; Moskovits, M.; Kneipp, H. Surface-enhanced raman scattering: physics and applications; Springer, 2006.

(31) Jensen, L.; Aikens, C. M.; Schatz, G. C. Electronic structure methods for studying surfaceenhanced Raman scattering. Chem. Soc. Rev. 2008, 37, 1061-1073.

(32) Fromm, D.; Sundaramurthy, A.; Kinkhabwala, A.; Schuck, P.; Kino, G.; Moerner, W. E. Exploring the chemical enhancement for surface-enhanced Raman scattering with Au bowtie nanoantennas. J. Chem. Phys. 2006, 124, 061101.

(33) Valley, N.; Jensen, L.; Autschbach, J.; Schatz, G. C. Theoretical studies of surface enhanced hyper-Raman spectroscopy: The chemical enhancement mechanism. J. Chem. Phys. 2010, 133, 054103.

(34) Saikin, S. K.; Olivares-Amaya, R.; Rappoport, D.; Stopa, M.; Aspuru-Guzik, A. On the chemical bonding effects in the Raman response: Benzenethiol adsorbed on silver clusters. Phys. Chem. Chem. Phys. 2009, 11, 9401-9411.

(35) Saikin, S. K.; Chu, Y.; Rappoport, D.; Crozier, K. B.; Aspuru-Guzik, A. Separation of Electromagnetic and Chemical Contributions to Surface-Enhanced Raman Spectra on Nanoengineered Plasmonic Substrates. J. Phys. Chem. Let. 2010, 1, 2740-2746.

(36) Zou, S.; Schatz, G. C. Silver nanoparticle array structures that produce giant enhancements in electromagnetic fields. Chemical Physics Letters 2005, 403, 62 - 67.

(37) Aikens, C. M.; Schatz, G. C. TDDFT Studies of Absorption and SERS Spectra of Pyridine Interacting with Au20. J. Phys. Chem. A 2006, 110, 13317-13324.

(38) Chen, H.; McMahon, J.; Ratner, M.; Schatz, G. Classical Electrodynamics Coupled to Quantum Mechanics for Calculation of Molecular Optical Properties: a RT-TDDFT/FDTD Approach. J. Phys. Chem. C 2010, 114, 14384-14392. 
(39) Masiello, D. J.; Schatz, G. C. Many-body theory of surface-enhanced Raman scattering. Phys. Rev. A 2008, 78, 042505.

(40) Chew, H.; Wang, D.; Kerker, M. Surface enhancement of coherent anti-Stokes Raman scattering by colloidal spheres. J. Opt. Sci. Am. B 1984, 1, 56-66.

(41) Ichimura, T.; Hayazawa, N.; Hashimoto, M.; Inouye, Y.; Kawata, S. Local enhancement of coherent anti-Stokes Raman scattering by isolated gold nanoparticles. J. Raman Spectrosc. 2003, 34, 651-654.

(42) Namboodiri, V.; Namboodiri, M.; Diaz, G. C.; Oppermann, M.; Flachenecker, G.; Materny, A. Surface-enhanced femtosecond CARS spectroscopy (SE-CARS) on pyridine. Vibrational Spectroscopy 2011, 56, 9-12.

(43) Jensen, L.; Zhao, L. L.; Schatz, G. C. Size-Dependence of the Enhanced Raman Scattering of Pyridine Adsorbed on Ag Clusters. J. Phys. Chem. C 2007, 111, 4756-4764.

(44) Zhao,; Jensen, L.; Schatz, G. C. Pyridine Ag-20 Cluster: A Model System for Studying Surface-Enhanced Raman Scattering. J. Am. Chem. Soc. 2006, 128, 2911-2919, PMID: 16506770.

(45) Foggi, P.; Salvi, P.; Bini, R.; Castellucci, E. author. J. Mol. Struc. 1988, 175, 147-152.

(46) Jensen, L.; Zhao, L. L.; Autschbach, J.; Schatz, G. C. Theory and method for calculating resonance Raman scattering from resonance polarizability derivatives. The Journal of Chemical Physics 2005, 123, 174110.

(47) Morton, S. M.; Jensen, L. Understanding the Molecule-Surface Chemical Coupling in SERS. Journal of the American Chemical Society 2009, 131, 4090-4098, PMID: 19254020.

(48) Zayak, A. T.; Hu, Y. S.; Choo, H.; Bokor, J.; Cabrini, S.; Schuck, P. J.; Neaton, J. B. Chemical Raman Enhancement of Organic Adsorbates on Metal Surfaces. Phys. Rev. Lett. 2011, 106, 083003. 
(49) Shuming, N.; Lipscomb, L. A. Surface-Enhanced Hyper-Raman Spectroscopy. Applied Spectroscopy Reviews 1991, 26, 203.

(50) Li, W.-H.; Li, X.-Y.; Yu, N.-T. Surface-enhanced hyper-Raman spectroscopy (SEHRS) and surface-enhanced Raman spectroscopy (SERS) studies of pyrazine and pyridine adsorbed on silver electrodes. Chemical Physics Letters 1999, 305, 303 - 310.

(51) Addison, C.; Konorov, S.; Brolo, A.; Blades, M.; Turner, R. Tuning Gold Nanoparticle SelfAssembly for Optimum Coherent Anti-Stokes Raman Scattering and Second Harmonic Generation Response. J. Phys. Chem. C 2009, 113, 3586-3592.

(52) Hetherington, W. Surface CARS spectroscopy of pyridine and phenol on ZnO optical waveguides. Chem. Phys. Lett. 1986, 128, 150-155.

(53) Kim, H.; Taggart, D. K.; Xiang, C.; Penner, R. M.; Potma, E. O. Spatial Control of Coherent Anti-Stokes Emission with Height-Modulated Gold Zig-Zag Nanowires. Nano Letters 2008, 8, 2373-2377, PMID: 18662040.

(54) Renger, J.; Quidant, R.; Hulst, N. V.; Novotny, L. Surface-Enhanced Nonlinear Four-Wave Mixing. Phys. Rev. Lett. 2010, 104, 046803.

(55) Roy, S.; Meyer, T. R.; Gord, J. R. Time-resolved dynamics of resonant and nonresonant broadband picosecond coherent anti-Stokes Raman scattering signals. Appl. Phys. Lett. 2005, $87,264103$.

(56) Pestov, D.; Zhi, M.; Sariyanni, Z.; Kalugin, N.; Kolomenskii, A.; Murawski, R.; Paulus, G.; Sautenkov, V.; Schuessler, H.; Sokolov, A. Visible and UV coherent Raman spectroscopy of dipicolinic acid. Proc. Nat. Acad. Sci. 2005, 102, 14976.

(57) Lucht, R. P.; Kinnius, P. J.; Roy, S.; Gord, J. R. Theory of femtosecond coherent anti-Stokes Raman scattering spectroscopy of gas-phase transitions. J. Chem. Phys. 2007, 127, 044316. 
(58) Dlott, D. D.; Schosser, C. L.; Chronister, E. L. Temperature-dependent vibrational dephasing in molecular crystals: a picosecond cars study of naphthalene. Chemical Physics Letters 1982, $90,386-390$.

(59) Schosser, C. L.; Dlott, D. D. A picosecond CARS study of vibron dynamics in molecular crystals: Temperature dependence of homogeneous and inhomogeneous linewidths. The Journal of Chemical Physics 1984, 80, 1394-1406.

(60) Head-Gordon, M.; Tully, J. C. Vibrational relaxation on metal surfaces: Molecular-orbital theory and application to $\mathrm{CO} / \mathrm{Cu}(100)$. J. Chem. Phys. 1991, 96, 3939.

(61) Guo, Z.; Habenicht, B. F.; Liang, W.; Prezhdo, O. Ab initio study of phonon-induced dephasing of plasmon excitations in silver quantum dots. Physical Review B 2010, 81, 125415.

(62) Cui, M.; Joffre, M.; Skodack, J.; Ogilvie, J. P. Interferometric Fourier transform Coherent anti-Stokes Raman Scattering. Opt. Express 2006, 14, 8448-8458.

(63) TURBOMOLE V6.2 2010, a development of University of Karlsruhe and Forschungszentrum Karlsruhe GmbH, 1989-2007, TURBOMOLE GmbH, since 2007; available from http://www.turbomole.com.

(64) Rappoport, D.; Furche, F. Analytical time-dependent density functional derivative methods within the RI-J approximation, an approach to excited states of large molecules. J. Chem. Phys. 2005, 122, 064105.

(65) Rappoport, D.; Furche, F. Lagrangian approach to molecular vibrational Raman intensities using time-dependent hybrid density functional theory. J. Chem. Phys. 2007, 126, 201104.

(66) Schäfer, A.; Huber, C.; Ahlrichs, R. Fully Optimized Contracted Gaussian Basis Sets of Triple Zeta Valence Quality for Atoms Li to Kr. J. Chem. Phys. 1994, 100, 5829.

(67) Becke, A. D. Density-functional thermochemistry. III. The role of exact exchange. The Journal of Chemical Physics 1993, 98, 5648-5652. 
(68) Perdew, J. P.; Ernzerhof, M.; Burke, K. Rationale for mixing exact exchange with density functional approximations. The Journal of Chemical Physics 1996, 105, 9982-9985.

(69) Adamo, C.; Barone, V. Toward reliable density functional methods without adjustable parameters: The PBE0 model. J. Chem. Phys. 1999, 110, 6158-6170. 